Für Patienten mit risikoreichem Alkoholkonsum sind Hausärztinnen und Hausärzte wichtige Ansprechpartner - wenn diese es denn tun und wenn sie auch wissen, wie es getan werden kann! Der erste Leitfaden, der die Ärztinnen und Ärzte bei dieser anspruchsvollen Aufgabe unterstützte, entstand im Rahmen des nationalen Alkoholpräventionsprogramms "Alles im Griff?» vor nunmehr zehn Jahren. Seither haben sich die therapeutischen Optionen deutlich verbessert, die Autor(inn)en des Leitfadens haben deshalb dankenswerterweise eine zweite, erweiterte Auflage des Leitfadens zur «Kurzinterventionen bei Patienten mit risikoreichem Alkoholkonsum» verfasst, die hier vorgestellt wird.

Dr. med. Christine Romann, Mitglied des Zentralvorstandes der FMH, Departementsverantwortliche Gesundheitsförderung und Prävention

\title{
Durch Kurzinterventionen zur «harm reduction»
}

\section{Pierre Loeb}

Dr. med., Facharzt für Allgemeinmedizin, Basel

\section{Alkoholprobleme - in der hausärztlichen Sprechstunde gern gemieden}

Dieses Vermeidungsverhalten hat mehrere Ursachen, eine hat damit zu tun, dass die bisherigen Erfolgsraten äusserst bescheiden sind, und welcher betreuende Arzt will seinem Patienten schon eine Suchtbehandlung oder gar einen Aufenthalt in einer «Entzugsanstalt» zumuten ... Dies mag etwas übertrieben klingen, doch bisher hatten wir wenig therapeutische Optionen, und für die gemäss Leitlinien anzuvisierende Abstinenz hat uns in den meisten Fällen der Glaube gefehlt. Auch alle anderen Formen von «kontrolliertem Trinken» wie: «Trinken Sie ein bisschen weniger ..., erst am Abend das erste Glas ..., und nie mehr als drei Einheiten ..., beginnen Sie mit Mineralwasser ..., versuchen Sie es mit einem alkoholfreien Bier ..., schieben Sie einen alkoholfreien Tag dazwischen ..., trinken Sie nie allein ..., nie zu Hause» usw. waren alles gutgemeinte aber selten mittel- oder langfristig erfolgversprechende Empfehlungen. Ähnlich geht es uns mit der autoritären Art mittels Drohungen: «Ihre Gamma-GT, Ihre Leberwerte sind erneut angestiegen, im Ultraschall finden sich Zeichen ...» Kommt dann noch hinzu, dass das Ansprechen heikler Themen eine gewisse Schwellenüberschreitung verlangt, die Beziehung zu unserem Patienten oft strapaziert und, last but not least, geniessen wir selber auch gern einen guten Tropfen und wissen genau, dass wir bei uns von Tropfen, beim Patienten aber von Dezilitern sprechen - kurz: wollen wir wirklich darauf eingehen, erfordert es viel Zeit und öffnet die Büchse der Pandora: sexuelle Probleme, Probleme bei der Arbeit, in der Ehe, häusliche Gewalt, finanzielle Konsequenzen - alles gute Gründe, das
Thema doch besser nicht anzuschneiden, wer weiss, was wir da für eine Lawine lostreten.

Vor zehn Jahren erschien der erste Leitfaden im Rahmen des nationalen Alkoholpräventionsprogamms "Alles im Griff?». Damals konzentrierte sich die Kampagne des Bundesamtes auf die Gruppe jugendlicher Risikotrinker. Mittels einer aufwendigen und sehr einprägsamen Wort- und Bildspiel-Aktion mit Whiskygläsern (Sie erinnern sich?) entwickelte die begleitende "Arbeitsgruppe Ärzte» für Hausärzte Workshops, um das Erkennen des Risikotrinkens - das primär nicht als pathologisch oder übertrieben wahrgenommen wurde - durch direktes Ansprechen und anhand klarer Vergleichszahlen im Sinne von «Wie viel ist zu viel?» zu erlernen. Zur Ergänzung und zum Selbststudium entstand die erste Ausgabe des Leitfadens, der eine bessere Selektions- und Erkennungstechnik vorstellte und dem behandelnden Arzt gleichzeitig auch eine Möglichkeit bot, wie er mit dem Patienten das Gespräch eröffnen und schon erste Schritte der Behandlung mittels der Kurzinterventionen einleiten konnte. Nicht eingeschlossen in diesem Behandlungskonzept waren jedoch chronisch alkoholabhängige Patienten, für die nur ein Entzug und die Abstinenzbehandlung als Option bestand.

\section{Aktualisierte und erweiterte Auflage des Leitfadens}

Was hat sich in den letzten zehn Jahren verändert und was hat die Autoren bewogen, gerade jetzt eine zweite, erweiterte Auflage herauszugeben? Drei wesentliche Punkte mögen dies hervorheben: Erstens: Heute, zehn Jahre später, ist harm reduction 


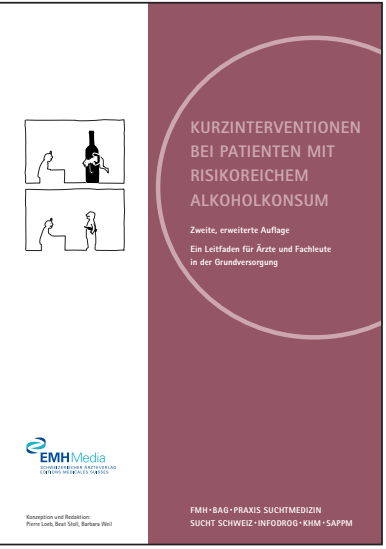

Pierre Loeb, Beat Stoll, Barbara Weil (Konzept, Redaktion) Kurzinterventionen bei Patienten mit risikoreichem Alkoholkonsum

Ein Leitfaden für Ärzte und Fachleute in der Grundversorgung Basel: EMH Schweizerischer Ärzteverlag; 2015.

2., erweiterte Auflage. 43 Seiten. 5 CHF. ISBN 978-3-03754o81-7 ein anerkannter Ansatz, der einem Paradigmenwechsel in der Alkoholbehandlung gleichkommt. So lässt sich die jüngst erschienene S3-Leitlinie «Screening, Diagnose und Behandlung alkoholbezogener Störungen» der Deutschen Gesellschaft für Psychiatrie, Psychosomatik und Nervenheilkunde (DGPPN) folgendermassen zusammenfassen: "Die Leitlinie empfiehlt nun auch die Reduktion des Alkoholkonsums als Therapieziel. Sie kann ein Zwischenschritt zur Abstinenz sein und gibt nach Meinung der Experten Anlass zur Hoffnung, Alkoholabhängige frühzeitiger zu einer Therapie zu bewegen»[1].

Zweitens: Neue Perspektiven werden nun auch durch die veränderten therapeutischen Möglichkeiten eröffnet. Das Aversions-Konzept, mit Disulfiram «das Trinken zu verderben», wich zugunsten neuer Substanzgruppen, wie der oralen Opioid-System-Modulatoren, die vor allem die Lust am vermehrten Alkoholkonsum reduzieren sollen.

Drittens: Diese Erweiterung der therapeutischen Möglichkeiten verleiht dem Arzt neue Möglichkeiten, seinen alkoholkranken Patienten zu begegnen, und dem Ansatz der Kurzintervention noch mehr Bedeutung, da diese medikamentöse Bedarfsbehandlung laut Swissmedic durch eine motivierende und adhärenzfördernde Intervention begleitet werden muss, um wirklich zu einem Erfolg zu führen. Dabei bleibt dem Hausarzt ganz klar eine Schlüsselrolle, wenn es darum geht, die Problematik anzusprechen und eine Behandlung anzuregen. Wenn es ein Arzt schafft, mittels Kurzinterventionen selbst zu behandeln oder jemanden zum Aufsuchen einer Beratungsstelle zu motivieren, ist schon sehr viel erreicht.

Dies ist mit ein Grund, weshalb mit der Markteinführung 2014 der oralen Opioidrezeptor-Modulatoren (z.B. Nalmefen) die «Kurzinterventionen bei Alkoholkonsum» zur Unterstützung der verordnenden Ärzte neu aufgelegt worden ist. Somit kann ein Wechsel von der ausschliesslich abstinenzorientierten Behandlung zur Schadensminderung harm reduction gefördert werden. Gewisse alkoholkranke Patienten zeigen sich eher bereit, an einem reduzierten Konsum zu arbeiten, als sich mit einer kompletten Abstinenz auseinanderzusetzen.

Weiter wurde die zweite Auflage mit einem Kapitel über die aktuell in der Schweiz im Handel befindlichen Medikamente ergänzt, die zur Unterstützung bei der Alkoholbehandlung zur Verfügung stehen und die inzwischen stark angewachsene neue Literaturliste ergänzt. Prof. A. Kiss, Chefarzt Psychosomatik, Universitätsspital Basel, fasst in einer kritischen Literaturübersicht die Entwicklung der letzten zehn
Jahre zusammen, und Abstracts ausgewiesener Arbeiten finden sich übersetzt im dritten Teil des Leitfadens "Bibliografie - das Wissen vertiefen". Viel Gewicht wurde auch darauf gelegt, den Leitfaden auf und mit den wichtigsten Webseiten, die in der Suchtmedizin aktiv sind, zu verlinken (www.fosumos.ch, www.safezone.ch, www.suchtschweiz.ch, www.info drog.ch, www.mydrinkcontrol.ch).

Das Kernstück des Leitfadens bilden aber die Kurzinterventionen, die das wichtigste Werkzeug des behandelnden Arztes sind und das Vorgehen bestimmen. Es schien den damaligen Autoren schon notwendig und nützlich, interessierten Personen neben der Fortbildung und den Sensibilisierungsprogrammen eine leicht lesbare Broschüre zur Verfügung zu stellen. Dieser Leitfaden ist in erster Linie für Ärzte bestimmt, und zwar sowohl für Grundversorger als auch für Fachärzte (Arbeitsmediziner, Militärärzte, Schulärzte, Zahnärzte, Gynäkologen, Sportärzte usw.). Der Leitfaden setzt sich aus einem praktischen und einem Erklärungsteil zusammen: Im praktischen Teil werden Schritt um Schritt die spezifische Aufgaben jedes Interventionsschritts der Kurzintervention beschrieben. Praktische Beispiele aus der Praxis erläutern die Schritte. Im Erklärungsteil werden Prinzipien und zugrundeliegende Konzepte im Detail erklärt. Der Leser findet zusätzlich die Referenzen zur Literatur und verschiedene technische Ansätze, die das Lernen erleichtern. Dies sollte helfen, dass jeder seine eigene Vorgehensweise und seinen persönlichen Stil entwickeln kann und dabei die zentralen Besonderheiten der Methode berücksichtigt. Im letzten Teil findet sich eine kommentierte Bibliographie, die die wichtigsten wissenschaftlichen Arbeiten kommentiert und versucht, auch kritische Fragen zu beantworten. Der neue Leitfaden hat auch bei anderen Fachleuten aus dem Sozial- und Gesundheitswesen Interesse geweckt, die aufgrund ihrer Arbeit Kontakt mit Risiko- und chronischen Trinkern haben: Sozialarbeitern, Pflegefachpersonen, Apothekern, Physiotherapeuten und Psychologen. Kritiker mögen das Potential der Kurzintervention als zu bescheiden bewerten. Dabei ist aber zu bedenken, dass bereits ein geringer Effekt, auf eine breite Bevölkerungsgruppe angewandt, am Ende einen bedeutenden Erfolg ergeben kann («Community Effectiveness»).

Der Leitfaden kann für 5 CHF (plus Porto und Verpackung) beim Sekretariat SAPPM, Postfach 521, 6260 Reiden, info[at]sappm.ch, bestellt werden.

1 www.awmf.org/uploads/tx_szleitlinien/o76-oo1l_S3-Leitlinie_ Alkohol_2015-01.pdf 